Research Article

\title{
Fractional-Order PID and Active Disturbance Rejection Control with Tuning Parameter Optimization for Induction Heating
}

\author{
Bingyu Li, ${ }^{1}$ Jining Guo $\mathbb{D}^{1,2}$ and Ying Fu ${ }^{1}$ \\ ${ }^{1}$ Bohai University, Jinzhou 121013, Liaoning, China \\ ${ }^{2}$ Northeast Forestry University, Harbin 150040, Heilongjiang, China \\ Correspondence should be addressed to Jining Guo; guojining1@163.com
}

Received 5 November 2021; Revised 22 November 2021; Accepted 27 November 2021; Published 20 December 2021

Academic Editor: Kauko Leiviskä

Copyright (c) 2021 Bingyu Li et al. This is an open access article distributed under the Creative Commons Attribution License, which permits unrestricted use, distribution, and reproduction in any medium, provided the original work is properly cited.

Induction heating systems are characterized by model uncertainty, nonlinearity, and external disturbances, and the control accuracy of the system directly affects the performance of the heated workpiece. In order to improve the temperature control accuracy and anti-interference performance of induction heating systems, this paper proposes a composite control strategy combining fractional-order PID (FOPID) and active disturbance rejection control (ADRC). Meanwhile, for the problem of too many controller tuning parameters, an improved quantum behavior particle swarm optimization (QPSO) algorithm is used to transform the nine parameters to be tuned in fractional-order PID active disturbance rejection control (FOPID-ADRC) into a minimization value optimization problem for solving. The simulation results show that the FOPID-ADRC controller improves the anti-interference capability and control accuracy of the temperature control system, and the improved QPSO algorithm has better global search capability and local optimal adaptation value.

\section{Introduction}

Vacuum induction melting technology is a kind of induction heating technology with high heating efficiency, high speed, and low consumption. It is developed and optimized on the basis of an ordinary induction furnace. With the development of the aerospace industry, nuclear energy, and other industries, sophisticated industrial equipment has higher requirements for material performance and smelting machinery. In view of the high performance and high precision requirements of the process refining process, a large number of scholars have found through theoretical discussion and practical research that the special smelting technology of high-precision materials in a vacuum environment can meet the performance requirements of the equipment to a certain extent. Alloys made in vacuum are widely used in cuttingedge technologies such as biotechnology (prosthetic implants) and aviation (turbine blades) [1].
There are not many existing studies on induction heating temperature control. The temperature control method used in induction heating furnaces in actual industrial production processes is the classical PID control. The temperature in the chamber is measured by thermocouples in the vacuum induction furnace, and when the measured temperature deviates from the set temperature, the heating input voltage is adjusted to minimize the deviation, which is a typical feedback control process [2]. However, with the development of cutting-edge technology and the increasing quality requirements for workpieces in various fields, induction heating temperature should be controlled using more precise control methods. The work in [3] applied fuzzy PID combined with expert control for heating furnace temperature control. This method improves the response speed of the temperature control system, but does not improve the antiinterference capability of the temperature control system. The work in [4] applies switching predictive control to the temperature control system of a heating furnace. This 
control strategy provides an effective and independent control mode for the temperature of the heating furnace, but relies on the conventional implementation of multiple model switching states, making the control algorithm too complex for application in engineering practices. In [5], an improved fractional-order predictive control is proposed and applied to an industrial heating furnace to improve the dynamic performance and stability of the temperature control system, but no parameter tuning method for the controller is provided. In [6], the improved Smith predictive control was applied to the outlet temperature control of the heater, and the control method improved the closed-loop stability when the process parameters of the heater changed, but the problem of disturbances caused by load changes and the requirement of high accuracy of the system were not well solved. The temperature control system of the vacuum induction furnace is shown in Figure 1.

Active disturbance rejection control (ADRC) is a new type of controller formed on the basis of the classical PID controller which does not depend on the precise mathematical model of the controlled object. The real-time disturbances of the system are observed by the extended state observer (ESO), and the control quantities are compensated so that the output of the system has better resistance to disturbances. ADRC is now widely used in areas such as motion systems, mechanical systems, motor systems, and marine system control [7-10]. To improve the control accuracy of ARDC, many scholars have introduced control methods such as sliding mode control, fuzzy control, backstepping control, and fractional-order PID (FOPID) control into the ADRC controller as the nonlinear state error feedback control law. Among them, FOPID is more easily applied in engineering practice because of its fewer control parameters and simple structure [11, 12]. In [13], the fractional calculus is introduced into ADRC, where the extended state observer (ESO) and the nonlinear state error feedback (NLSEF) are replaced by the fractional-order extended state observer and PID controller, respectively. Robustness analysis shows that FADRC is also appropriate for an incommensurate fractional-order system. In [14], a fractional-order PID and active disturbance rejection controller (FOPID-ADRC) is applied to the speed regulation system of the nonlinear double-mass servo drive system, and it is proved that the compound controller has good robustness. In [15], the FOPID-ADRC is applied to the tracking control of an air-breathing hypersonic vehicle, and the simulation results prove that the designed controller has good adaptability and robustness in dealing with uncertainty and external disturbance. In this paper, a fractional-order PID active disturbance rejection control (FOPID-ADRC) strategy is used to control the temperature in the induction heating process, and an improved QPSO is adopted to rectify the parameters of the controller in order to improve the control accuracy as well as the antidisturbance capability of the temperature control system.

The main research of this paper is as follows: Section 1 is the introduction; Section 2 briefly describes the principle of the vacuum induction furnace and its mathematical model; Section 3 introduces the design of FOPID-ADRC; Section 4 shows the invocation of the improved QPSO optimization method; Section 5 is the simulation experiment and analyzes the anti-interference and control accuracy of FOPID-ADRC; and Section 6 is the conclusion.

\section{Mathematical Model of an Induction Furnace}

The induction heating furnace is a nonlinear system with large inertia and large time lag. The main phenomena involved in the heating process are the skin effect and heat conduction. When the induction heating coil passes an alternating current, an alternating magnetic field is generated in and around the coil, and the workpiece is cut by the magnetic lines of force formed by the alternating magnetic field $[16,17]$. According to the electromagnetic field theory, the induced electric potential generated by a changing magnetic field creates a closed circuit of current on the surface of the workpiece, which is usually referred to as eddy currents. The eddy currents generate heat inside the workpiece, causing it to be heated [18]. The schematic diagram of induction heating is shown in Figure 2.

The relationship between the thermal power and the surface temperature during the skin collection effect is [19]

$$
q_{w}=c m_{1} \frac{\Delta T_{2}}{\Delta t}+S q
$$

where $c$ is the specific heat capacity of the workpiece, $m$ is the mass of the heated part of the workpiece, $\Delta T_{2}$ is the temperature difference from the initial temperature at the completion of the heating phase, $\Delta t$ is the time required for the heating process, and $S$ is the surface area of the workpiece. $q$ is the heat lost per unit area of the workpiece surface. $q$ is linearly related to the temperature, and $q=k_{1} T$, where $k_{1}$ is the scale factor.

$$
q_{w}=c m \frac{\Delta T_{2}}{\Delta t}+S k_{1} T
$$

Taking Laplace transformations on both sides of equation (2), it is obtained that

$$
q_{w}(s)=c m s T_{s}+S k_{1} T(s) .
$$

Treating the specific heat capacity $c$ of the material as a constant, the process of the skin effect of the induction heating system can be obtained as an inertial link. The relationship between the heat-generating power $q_{w}$ and its surface temperature $T_{w}$ is

$$
\frac{T_{w}(s)}{q_{w}(s)}=\frac{K}{T_{m} s+1},
$$

where $K=\left(1 / S k_{1}\right), T_{m}=\left(\mathrm{cm} / S k_{2}\right)$, and $k_{2}$ is the scale factor. The transfer function of the heating process can be expressed as

$$
G_{0}(s)=\frac{K}{T_{m} s+1} .
$$

Since the heat transfer in the heat conduction process stage of the material is mainly transferred through the mutual collision movement between the molecules of the 


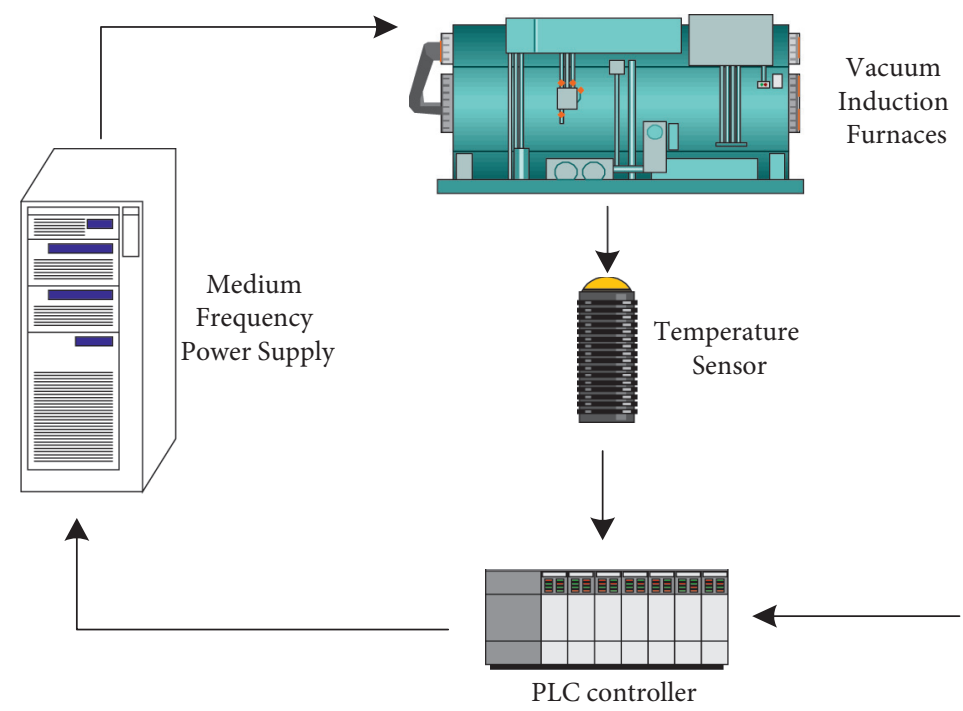

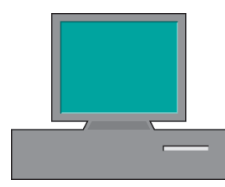

Upl ink

FIgURE 1: Temperature control system of the vacuum induction furnace.

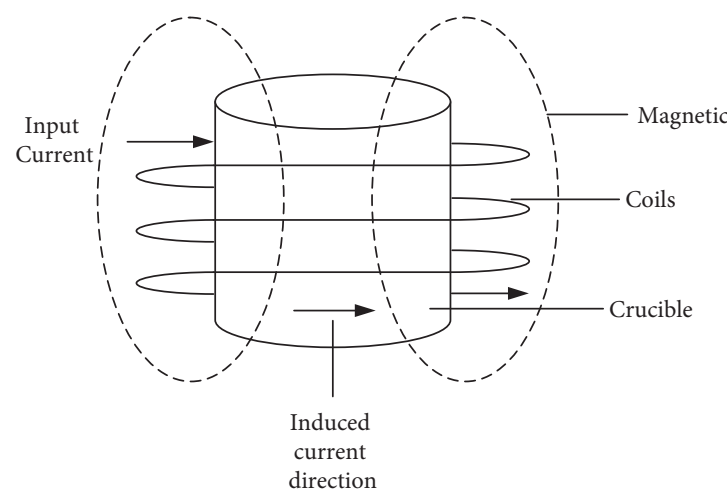

Figure 2: The schematic diagram of induction heating.

heating material, the induction heating of the heating material also takes a certain amount of time. Therefore, there is a process of heat generation and transfer, which causes a time delay between the input responses. Thus, the temperature control system in the vacuum induction furnace can be expressed as

$$
G(s)=\frac{K}{T_{m} s+1} e^{-\tau s},
$$

where $\tau$ is the time lag coefficient.

For the time lag term appearing in the system, the method of increasing the order can be adopted. In the transfer function, the time lag operator is approximated as a first-order inertia link.

$$
e^{-\tau s}=\frac{1}{1+\tau s}
$$

Thus, the temperature control system can be expressed as a second-order transfer function.

$$
G(s)=\frac{K}{\left(T_{m} s+1\right)(\tau s+1)} .
$$

The differential equation form of the system under the action of control law and perturbations can be expressed as

$$
\ddot{y} T_{m} \tau+\dot{y}\left(T_{m}+\tau\right)+y=K u+w,
$$

where $w$ is the total disturbance to the system and $u$ is the control law of the system. Then, the equation of state of the system can be expressed as

$$
\left\{\begin{array}{l}
\dot{x}_{1}=x_{2}, \\
\dot{x}_{2}=-\frac{T_{m}+\tau}{T_{m} \tau} x_{1}-\frac{1}{T_{m} \tau} x_{2}+\frac{K}{T_{m} \tau} u+w .
\end{array}\right.
$$

\section{Design of FOPID-ADRC}

3.1. FOPID. FOPID is developed on the basis of the classical PID. In order to control the dynamic system, Oustaloup 
proposed the fractional-order controller [20-22] and demonstrated that the performance of the controller was better than that of traditional PID. Later, Podlubny proposed the FOPID controller as a generalized form of the standard PID controller [23]. The FOPID controller has two free variables of integral order and differential order, which realizes the control from point to surface. Therefore, FOPID has a larger adjustable range and better control quality than traditional PID [24, 25]. is [26]

The Riemann-Liouville definition of fractional calculus

$$
{ }_{t_{0}}^{R L} D_{t}^{-\alpha} f(t)=\frac{1}{\Gamma(\alpha)} \int_{t_{0}}^{t} \frac{f(\tau)}{(t-\tau)^{1-\alpha}} \mathrm{d} \tau,
$$

where $D_{t}^{\alpha}$ is the fractional-order operator and $\Gamma(\alpha)$ is the gamma function.

The first derivative $D^{1} f(t)$ of $f(t)$ is defined as [26]

$$
D^{1} f(t)=\frac{\mathrm{d} f(t)}{\mathrm{d} t}=\lim _{h \longrightarrow 0} \frac{f(t)-f(t-h)}{h} .
$$

It can be seen that the fractional derivative is of great importance for long-term conditions. However, the integral derivative is only applicable to the current moment. The characteristic of the fractional derivative makes the system respond slowly and sensitive to disturbance [27].

The Laplace transform of Riemann-Liouville definition of fractional calculus is equal to

$$
L\left\{{ }_{0} D_{t}^{\alpha} f(t)\right\}=s^{\alpha} F(s)-\sum_{k=0}^{n-1} s^{k}\left[{ }_{0} D_{t}^{\alpha-k-1} f(t)\right]_{t=0} .
$$

Under zero initial conditions, the summation term in equation (4) is zero.

In general, a fractional-order system with one input and one output can be expressed as

$$
\begin{aligned}
& a_{n} y^{\left(\alpha_{n}\right)}(t)+a_{n-1} y^{\left(\alpha_{n-1}\right)}(t)+\cdots+a_{1} y^{\left(\alpha_{1}\right)}(t)+a_{0} y(t) \\
= & b_{m} u^{\left(\beta_{m}\right)}(t)+b_{m-1} u^{\left(\beta_{m}-1\right)}(t)+\cdots+b_{1} u^{\left(\beta_{1}\right)}(t)+b_{0} u(t) .
\end{aligned}
$$

The transfer function can be expressed as [27]

$$
G(s)=\frac{B(s)}{A(s)}=\frac{b_{m} s^{\beta_{m}}+b_{m-1} s^{\beta_{m}-1}+\cdots+b_{1} s^{\beta_{1}}+b_{0} s}{a_{n} s^{\alpha_{n}}+a_{n-1} s^{\alpha_{n-1}}+\cdots+a_{1} s^{\alpha_{1}}+a_{0}} .
$$

The transfer function of FOPID can be expressed as

$$
G_{\mathrm{FOPID}}(s)=\left(K_{p}+\frac{K_{i}}{s^{\lambda}}+K_{d} s^{\mu}\right) C(s) .
$$

The differential equation of FOPID can be expressed as

$$
u_{\mathrm{FOPID}}(t)=K_{p} c(t)+K_{i} D_{t}^{-\lambda} c(t)+K_{d} D_{t}^{\mu} c(t)
$$

where $u(t)$ is the controller output, $c(t)$ is the controller input, $\lambda$ and $\mu$ are any real numbers greater than zero, and $K_{p}, K_{i}$, and $K_{d}$ are the proportional gain, integral coefficient, and differential coefficient of the controller, respectively.
Reasonable selection of control parameters can obtain better dynamic performance and improve the control effect of the system. The schematic diagram of the FOPID controller can be expressed as in Figure 3.

3.2. Design of an FOPID-ADRC Composite Controller. ADRC consists of a tracking differentiator (TD), extended state observer (ESO), and nonlinear state error feedback controller (NLSEF) [28]. The transition process $v_{1}$ and the differential signals of each order of $v_{1}$ of system input $v$ can be obtained by using a tracking differentiator. According to the input and output data, the extended state observer can obtain the estimated values of the system state variables $z_{1} \sim z_{n}$ and the total disturbance of the system $z_{n+1}$. The nonlinear error feedback calculates the control signal $u_{0}$ according to the state error $e_{1} \sim e_{n}$ of the system [28]. $b_{0}$ is the controller gain, and $\omega$ is the external disturbance. ESO is the core module of ADRC, which is used to estimate and compensate the total disturbance in real time. The structural block diagram of ADRC is shown in Figure 4.

In the second-order FOPID-ADRC system, TD can be expressed as [28]

$$
\left\{\begin{array}{l}
\dot{x}_{1}=x_{2}, \\
\dot{x}_{2}=-r \operatorname{sign}\left(x_{1}-v(t)+\frac{x_{2}\left|x_{2}\right|}{2 r}\right),
\end{array}\right.
$$

where $r$ is the parameter to adjust the tracking speed. The larger the value of $r$, the faster the tracking speed. sign is a symbolic function. Since $\dot{x}_{1}=x_{2}, x_{2}$ can be approximately estimated as the derivative of the input signal. In the secondorder FOPID-ADRC, ESO as the core of the controller will expand the disturbance of the output of the controlled object into new variables $z_{3}$. The internal disturbance and external disturbance of the system are regarded as the total disturbance of the system, and a special feedback system is used to build the state that can be expanded, and then, the state of the system and all the disturbances are estimated in real time to give some compensation to the system. For a general second-order system, ESO can be expressed as [28]

$$
\left\{\begin{array}{l}
e=z_{1}-y_{1}, \\
\dot{z}_{1}=z_{2}-\beta_{1} g_{1}(e), \\
\dot{z}_{2}=z_{3}-\beta_{2} g_{2}(e), \\
\dot{z}_{3}=-\beta_{3} g_{3}(e),
\end{array}\right.
$$

where $z_{1}, z_{2}$, and $z_{3}$ are the observed values of $x_{1}, x_{2}$, and $x_{3}$, respectively, $x_{3}$ is the total disturbance of the system; and $\beta_{1}, \beta_{2}$, and $\beta_{3}$ are the gain of ESO, which can influence the strength of the observed signal. When the value is selected appropriately, the state of the system can be accurately estimated. $g_{1}, g_{2}$, and $g_{3}$ are the nonlinear functions which can be expressed as [29] 


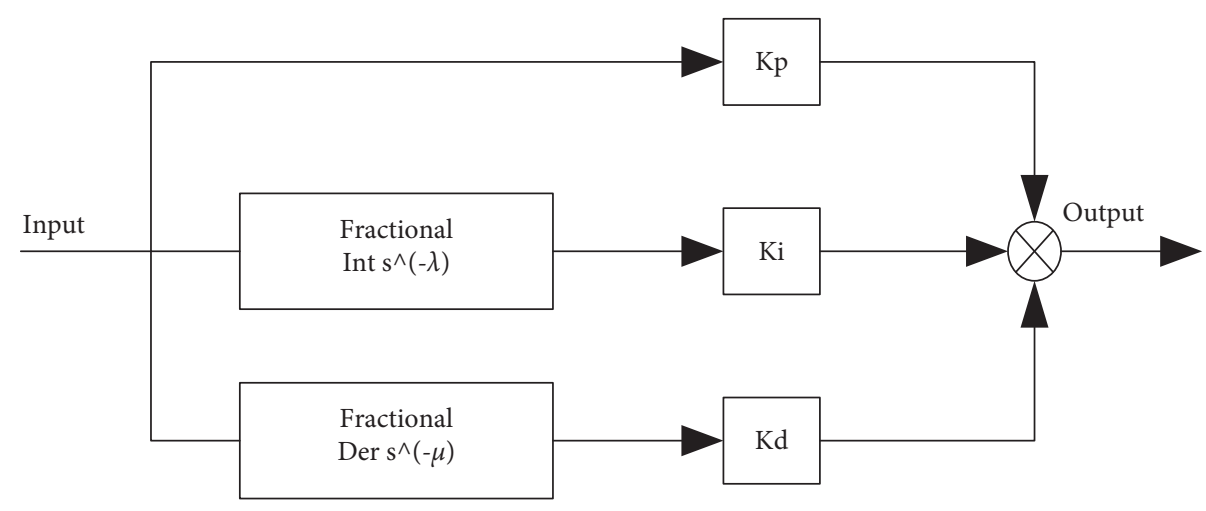

Figure 3: Schematic diagram of the FOPID controller.

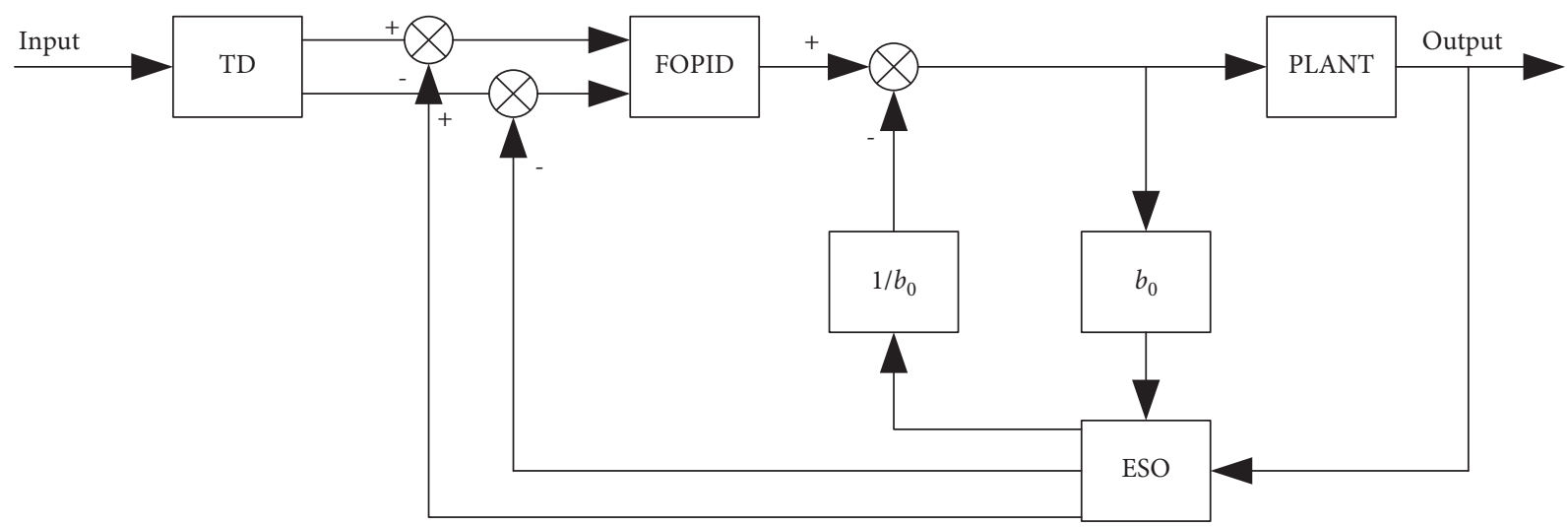

FIgure 4: The structural block diagram of the second-order ADRC.

$$
f a l(e, \alpha, \delta)= \begin{cases}\frac{e}{\delta^{1-\alpha}}, & |e|<\delta, \\ |e|^{\alpha} \operatorname{sign}(e), & |e|>\delta,\end{cases}
$$

where $\alpha$ and $\delta$ are adjustable parameters. If $\alpha=1$, the value of the nonlinear function is $e$. We can get the traditional Luenberger observer, also known as the linear observer, which is a special case of the nonlinear extended state observer [30]. There is coupling between $\alpha$ and $\delta$. If the value of $\delta$ is too large, the error range will become larger, and if the value of $\delta$ is too small, the output value of the error function will be too large. If the value of $\alpha$ is too small, it may lead to a high-frequency tremor of the control quantity. If the value of $\alpha$ is too large, it cannot play the advantages of fast error attenuation and strong disturbance rejection. Therefore, the values of $\alpha$ and $\delta$ are generally fixed, and the value of $\alpha$ is 0.25 or 0.5 , and the value of $\delta$ is 0.1 .

The output of ESO is $z_{1}, z_{2}$, and $z_{3}$. Thus, the control law can be expressed as

$$
u=\frac{-z_{3}+u_{0}}{b_{0}}
$$

where $b_{0}$ is the estimated value of $b . u_{0}$ is the virtual control law.
In this paper, the FOPID controller is introduced into the ADRC controller as a linear state error feedback controller. Therefore, the virtual control law $u_{0}$ can be expressed as [31]

$$
u_{0}=K_{p}\left(e_{1}-z_{1}\right)+\frac{K_{i}}{s^{\lambda}}\left(e_{2}-z_{2}\right)+K_{d} s^{\mu}\left(e_{2}-z_{2}\right) .
$$

\section{Parameter Tuning Method of Improved QPSO-Based FOPID-ADRC}

The commonly used setting method in ADRC is the empirical method proposed in the paper [32]. However, when there are too many control parameters, the empirical method often cannot obtain ideal dynamic performance. The QPSO algorithm is an optimization algorithm based on group intelligence theory. The basic principle of the algorithm is to establish a search mechanism by simulating the movement of a particle in a potential field toward the lowest point of potential energy in quantum mechanics [33]. Due to the superior performance of the quantum particle swarm algorithm, it can be used to solve for controller parameters.

4.1. Improved QPSO Algorithm. For the one-dimensional optimization problem, the position equation of particle $i$ is obtained by the Monte Carlo stochastic simulation method: 


$$
\begin{aligned}
X_{i}(t+1) & =p_{i}(t) \pm \frac{L_{i}(t)}{2} \ln \left(\frac{1}{\mu}\right), \\
L_{i}(t+1) & =2 \gamma(t)\left|C(t)-X_{i}(t)\right|, \\
C(t) & =\sum_{i=1}^{M} \frac{P_{B i}}{M}
\end{aligned}
$$

where $L(t)$ is the characteristic length of the potential well, $C(t)$ is the average optimal position of particles, $M$ is the total number of particles, $\gamma$ is the contraction-expansion coefficient which decides the rate of convergence of the algorithm, and $\gamma$ is defined as

$$
\gamma(t)=1-0.5 \times \frac{t}{T}
$$

where $t$ is the number of the current iteration and $T$ is the number of total iterations.

$p_{i}(t)$ is the local attractor, which is a random position between the optimal position of the first particle and the global optimal position. It is defined as [33]

$$
\begin{aligned}
p_{i}(t) & =\phi P_{B i}(t)+(1-\phi) P_{g}(t), \\
\phi & =\frac{c_{1} \operatorname{rand}_{1}}{c_{1} \operatorname{rand}_{1}+c_{2} \operatorname{rand}_{2}},
\end{aligned}
$$

where $c_{1}$ and $c_{2}$ are the learning factors. $c_{1}$ is used to adjust the step length of the particle to fly to its best position, reflecting the individuality of the particle. $c_{2}$ is used to adjust the step length of particles flying to the best position in the whole world, which reflects the sociality of particles. $P_{B i}(t)$ is the optimal position of the particle at the $t^{\text {th }}$ iteration, representing the global optimal position of the particle at the $\mathrm{t}^{\text {th }}$ iteration. rand is a random number between 0 and 1 [33].

As can be seen from equation (24), the contractionexpansion coefficient in the traditional QPSO algorithm has a linear relationship with the change of the number of iterations, which can meet the demand of global fast search at the initial stage of the search. However, when the particle is near the optimal value in the later searching period, the change rate of the contraction-expansion coefficient is constant, which makes the particle unable slow down the updating speed to carry out detailed searching. With the increase of the running time of the algorithm, the search particle may exceed the optimal value, resulting in the failure to achieve a good search effect.

Aiming at the problem that the traditional QPSO algorithm cannot search carefully in the late iteration, the work in [34] proposes to improve the relationship between the scaling coefficient and the number of iterations. The specific form of the improvement is shown as

$$
\gamma(t)=1-\frac{\cos (1-(t / T)) \pi}{2}
$$

Compared with equation (24), in the improved QPSO algorithm, the contraction-expansion coefficient has a nonlinear relationship with the current iteration number of the algorithm. As the number of iterations increases, the change rate of $\beta$ will obviously decrease. This change effectively avoids the situation that the evolution speed of particles exceeds the optimal value too fast and then enables particles to search carefully around the optimal value to find the optimal value. The update of particle position can be expressed as

$$
X_{i}(t+1)=p_{i}(t) \pm \beta(t)\left|C(t)-X_{i}(t)\right| \ln \left(\frac{1}{u}\right)
$$

The particle position in the improved QPSO will be adjusted continuously according to equation (27) until the termination condition is met or the global optimal solution is found [33].

4.2. Application of QPSO. The FOPID-ADRC has 11 parameters $\left\{r, h, K_{p}, K_{i}, K_{d}, \lambda, \mu, b_{0}, \beta_{1}, \beta_{2}, \beta_{3}\right\}$ that need to be tuned, which all affect the dynamic performance of the controller. $K_{p}$ is used to adjust the rapidity, $K_{i}$ is used to coordinate the stability of the system, and $K_{d}$ is used as the overshoot setting. $\lambda$ can improve the dynamic performance of the system while giving consideration to both the steadystate and dynamic performance of the system. $\mu$ can increase the damping of the system [5]. $b_{0}$ is a special controller parameter of ADRC. Different values of $b_{0}$ are equivalent to the total disturbance value changing in different ranges, and the compensation component will also change accordingly [25]. $\beta_{1}, \beta_{2}$, and $\beta_{3}$ are the gain of the ESO, and the values of these three will affect the observation effect of the extended state observer. $r$ is the speed factor in the TD, which determines the tracking speed of the signal. If the value of $r$ is appropriate, the system can reach the stable value quickly and without oscillation. $h$ is the integral step in the TD. Adjusting the value of $h$ can make the controller track the input signal without overshoot. The tracking differentiator works well when $r=1$ and $h=0.1$.

To sum up, the parameters that need to be set in FOPIDADRC are $\left\{K_{p}, K_{i}, K_{d}, \lambda, \mu, b_{0}, \beta_{1}, \beta_{2}, \beta_{3}\right\}$. To avoid instability in the control system, the size of the 9 parameters needs to be coordinated as accurately as possible. The QPSO algorithm is utilized to tune those parameters.

In order to obtain satisfactory control performance of the system, the rapidity, stability, and accuracy of the system are considered comprehensively. The overshoot $\sigma$, system error $e(t)$, and control quantity $u$ are integrated into the performance index in different forms. The fitness function selected in this paper is

$$
J=\int_{0}^{\infty}\left(K_{1} t|e(t)|+K_{2} u^{2}(t)\right) \mathrm{d} t+K_{3} \sigma,
$$

where $K_{1}, K_{2}$, and $K_{3}$ are the weight coefficients, selected according to experience, $K_{1}=K_{2}=1$ and $K_{3}=10$.

The optimization steps of the QPSO algorithm are shown as follows:

Step 1: the initial parameters of the algorithm are set, and the position and speed of the individual are initialized. 


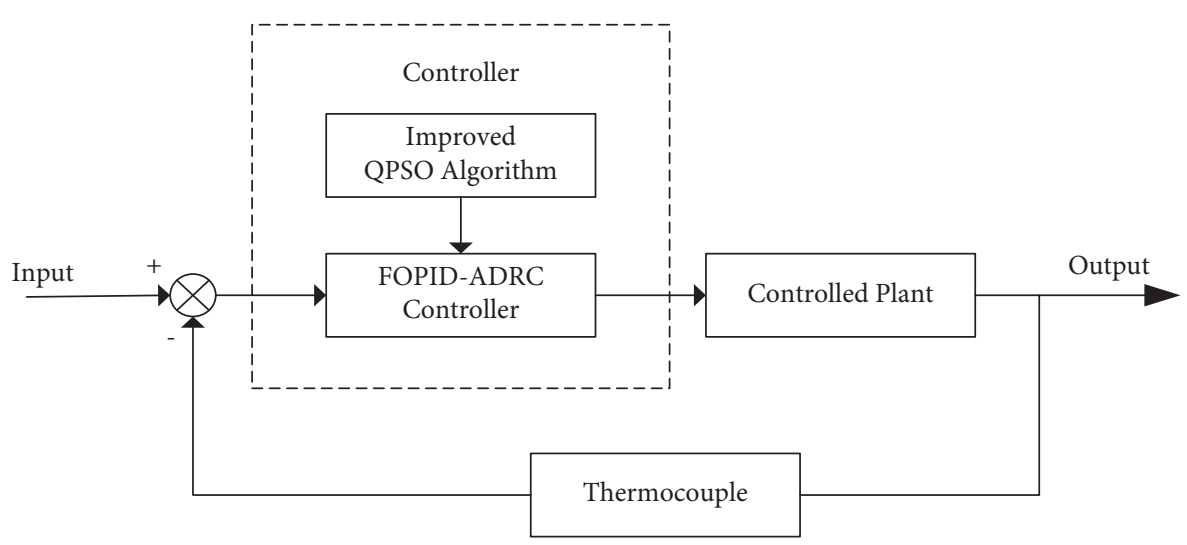

FIGURE 5: Improved QPSO algorithm for parameter optimization.

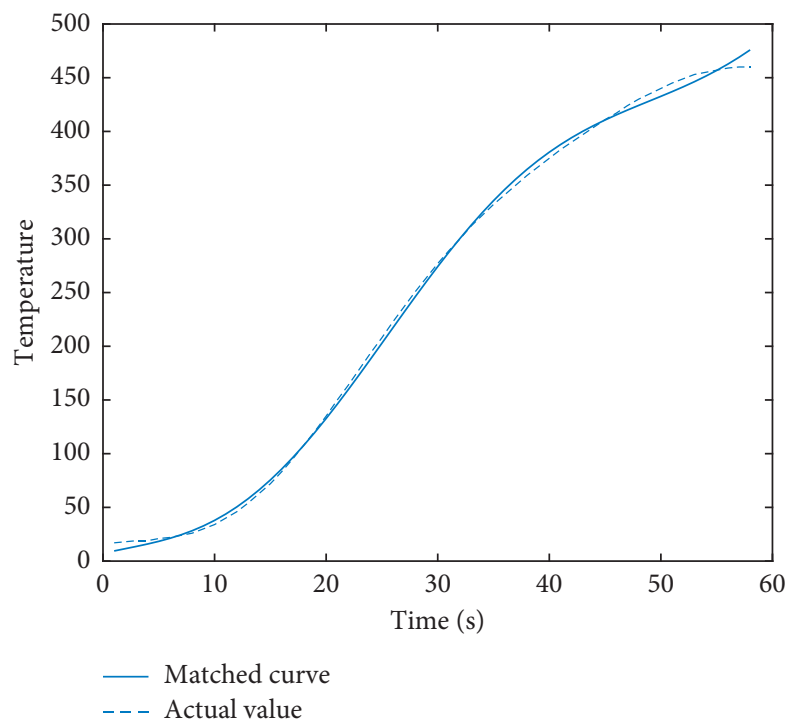

Figure 6: Data fitting results.

Step 2: the SIMULINK file is called, and the parameters to be adjusted in the algorithm program are passed to the FOPID-ADRC controller in turn. Then, the control system model is run. The fitness value is output and passed to the QPSO algorithm program. Step 3: the initial optimal individual particle and the initial global optimal particle are determined by ordering the initial fitness value extremum.

Step 4: the particle update operation is performed, and then, the part of Step 2 is invoked to calculate the fitness value to update the optimal particle.

Step 5: finally, according to whether the fitness value meets the condition or whether the maximum number of iterations is reached, if the condition is satisfied, we quit the algorithm, and the optimal solution set is obtained. If not, we proceed to Step 4.

The QPSO algorithm is used for parameter tuning, and its control structure is shown in Figure 5.

\section{Simulation Analysis}

A vacuum induction furnace is taken as the experimental object. When the input frequency of the induction furnace was set as $18863 \mathrm{~Hz}$, the change of its temperature is recorded every 30 seconds. The collected data were used for system identification in MATLAB, and the second-order system is shown in equation (29). The fitting result is shown in Figure 6.

$$
G_{\text {plant }}(s)=\frac{0.16}{s^{2}+0.06 s+0.02}
$$

Figure 7 shows the individual optimal iteration diagrams of FOPID-ADRC, compared with QPSO and improved QPSO parameter optimization in the case that the input is step response. The minimum value found by using the QPSO algorithm is about 18 , while the minimum value found by the improved QPSO algorithm is about 10. Therefore, it can be seen that the improved QPSO algorithm has better optimization ability. 


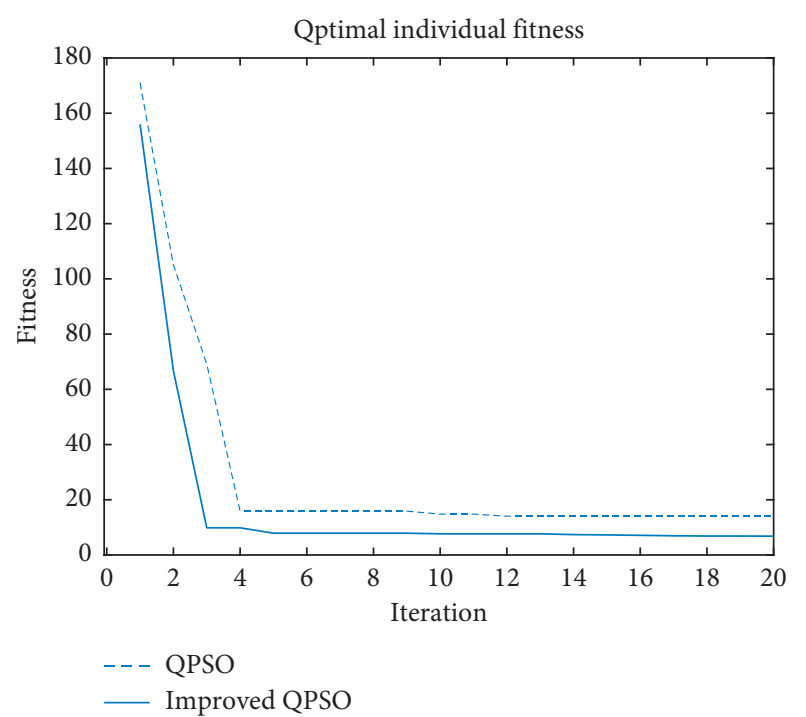

FIGURE 7: The iterative graphs of FOPID-ADRC parameters were optimized by QPSO and improved QPSO algorithms.

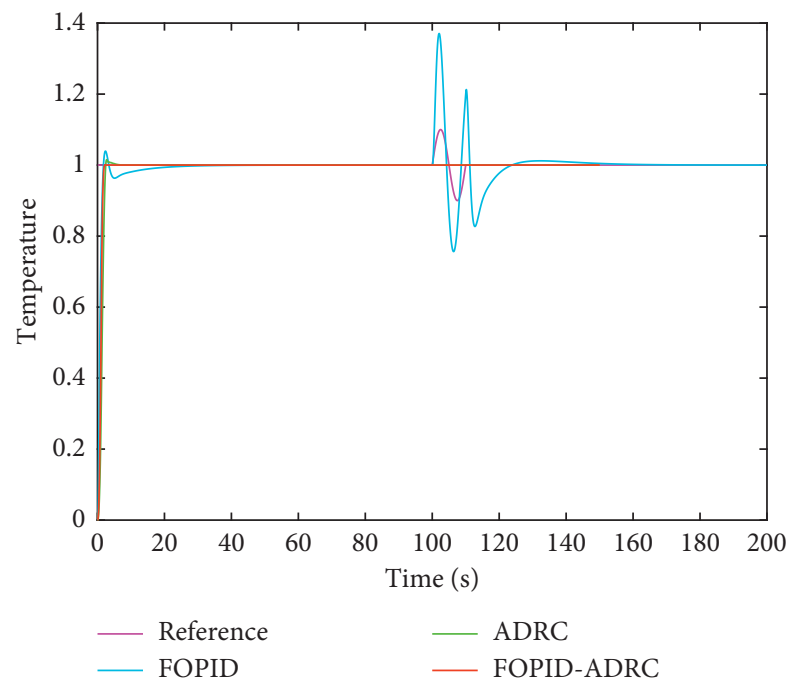

FIGURE 8: Output comparison of FOPID, ADRC, and FOPIDADRC when the input is step response.

Equation (29) is taken as the controlled system. When the input of the system is the step response, the simulation results and dynamic performance data are as shown in Figure 8. It can be seen that FOPID has small overshoot in the rising phase and the longest settling time. FOPID-ADRC has shorter stabilization time with no overshoot. When there is an external interference, FOPID-ADRC can maintain the stability of the system without the influence of the external interference.

The reference signal in Figure 9 shows the temperature rise to $100^{\circ} \mathrm{C}$ in the vacuum induction furnace and a disturbance of size 5 whose simulation of power disturbance in the induction furnace is added at $150 \mathrm{~s}$. It can be seen that when FOPID is used for control, the overshoot is the largest and the stability time is the longest. However, the overshoot of FOPID-ADRC is the lowest, and the stability time is shorter.

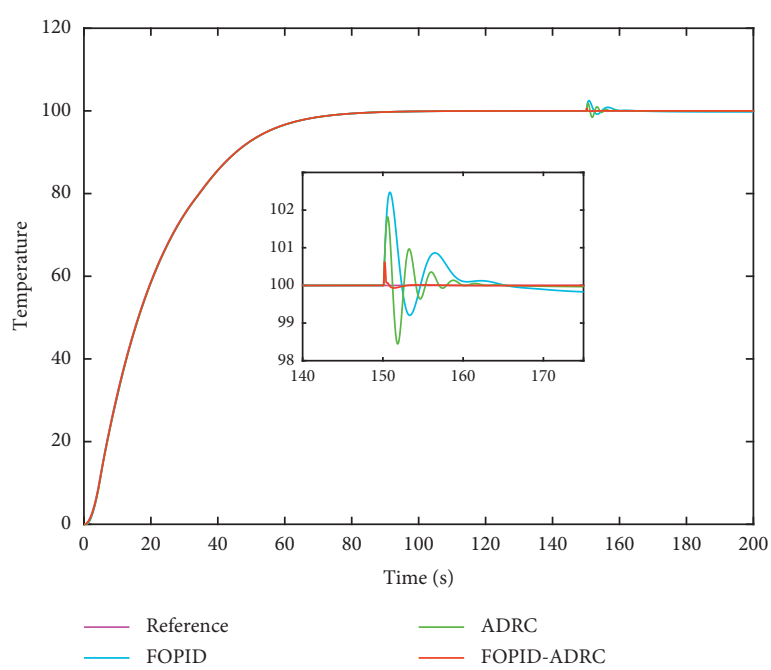

Figure 9: Comparison of control effects of FOPID, ADRC, and FOPID-ADRC when power disturbance is added.

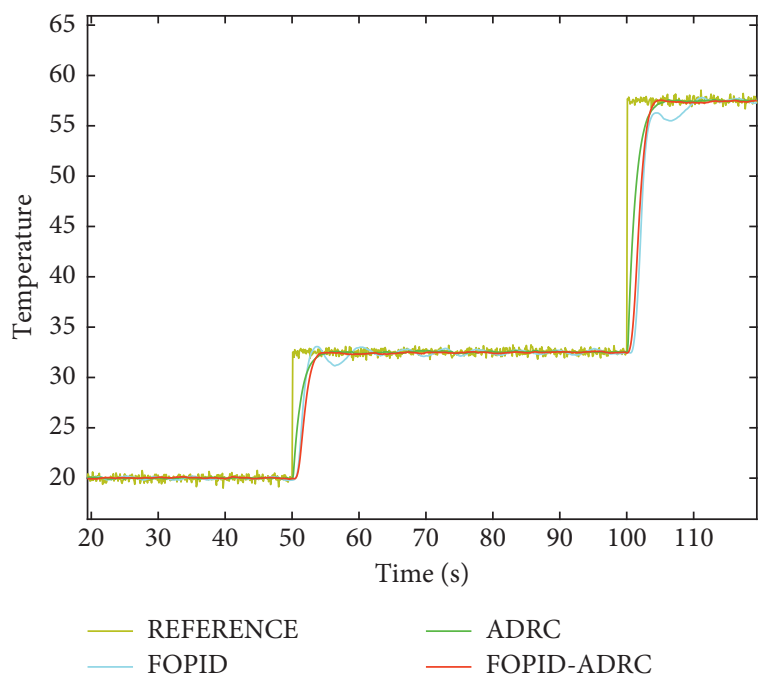

FIgURE 10: Comparison of control effects of FOPID, ADRC, and FOPID-ADRC when changing temperature order.

To verify the antidisturbance performance of the designed controller, Figure 10 shows the control effect of the three controllers when the reference temperature jumps at $50 \mathrm{~s}$ and $100 \mathrm{~s}$, respectively, using Gaussian white noisesimulated external disturbances and uncertainty of the model. It can be seen that the dynamic performance of FOPID-ADRC has the best antidisturbance performance compared to the other two controllers.

\section{Conclusions}

In this paper, the control principles of ADRC and FOPID are studied, and a composite control strategy is proposed and a FOPID-ADRC controller is designed for the unknown interference problem in the induction heating process. The simulation studies of ADRC, FOPID, and FOPID-ADRC under different operating conditions show that ADRC has 
higher antiturbulence capability compared with FOPID. FOPID-ADRC combines the advantages of both strategies to improve the control accuracy of the system while ensuring the anti-interference capability of the induction heating temperature control system.

For the problem of parameter tuning, an improved QPSO algorithm is used to optimize several parameters in the controller. The improved QPSO has better global search capability and local optimal fitness value compared to QPSO and also improves the efficiency of parameter tuning of the composite controller.

Moreover, the composite controller combining fractional PID and active disturbance rejection control is more complex than the PID controller adopted by conventional temperature control systems, and given the uncertainty in actual industrial production processes, further studies on the application of this controller in actual systems can be followed.

\section{Data Availability}

The raw data required to reproduce these findings cannot be shared at this time as the data also form part of an ongoing study.

\section{Conflicts of Interest}

The authors declare no conflicts of interest.

\section{References}

[1] P. Buliński, J. Smolka, S. Golak et al., "Numerical and experimental investigation of heat transfer process in electromagnetically driven flow within a vacuum induction furnace," Applied Thermal Engineering, vol. 124, pp. 1003-1013, 2017.

[2] X. Hao, J. Gu, N. Chen, W. Zhang, and X. Zuo, "3-D numerical analysis on heating process of loads within vacuum heat treatment furnace," Applied Thermal Engineering, vol. 28, no. 14-15, pp. 1925-1931, 2008.

[3] S. Dequan, G. Guili, G. Zhiwei, and X. Peng, "Application of expert fuzzy PID method for temperature control of heating furnace," Procedia Engineering, vol. 29, pp. 257-261, 2012.

[4] W. Xu, J. Zhang, and R. Zhang, "Application of multi-model switching predictive functional control on the temperature system of an electric heating furnace," ISA Transactions, vol. 68, pp. 287-292, 2017.

[5] R. Zhang, Q. Zou, Z. Cao, and F. Gao, "Design of fractional order modeling based extended non-minimal state space MPC for temperature in an industrial electric heating furnace," Journal of Process Control, vol. 56, pp. 13-22, 2017.

[6] V. Feliu-Batlle and R. Rivas-Perez, "Control of the temperature in a petroleum refinery heating furnace based on a robust modified Smith predictor," ISA Transactions, vol. 112, pp. 251-270, 2021.

[7] R. Garrido and L. Luna, "Robust ultra-precision motion control of linear ultrasonic motors: a combined ADRCLuenberger observer approach," Control Engineering Practice, vol. 111, Article ID 104812, 2021.

[8] R. Madonski, M. Ramirez-Neria, M. Stanković et al., "On vibration suppression and trajectory tracking in largely uncertain torsional system: an error-based ADRC approach,"
Mechanical Systems and Signal Processing, vol. 134, Article ID 106300, 2019.

[9] Y. Xu, W. Bai, S. Zhao, J. Zhang, and Y. Zhao, "Mitigation of forced oscillations using VSC-HVDC supplementary damping control," Electric Power Systems Research, vol. 184, Article ID 106333, 2020.

[10] Z. Chen, B. Qin, M. Sun, and Q. Sun, "Q-learning-based parameters adaptive algorithm for active disturbance rejection control and its application to ship course control," Neurocomputing, vol. 408, pp. 51-63, 2020.

[11] N. Nasirpour and S. Balochian, "Optimal design of fractionalorder PID controllers for multi-input multi-output (variable air volume) air-conditioning system using particle swarm optimization," Intelligent Buildings International, vol. 9, no. 2, pp. 107-119, 2017.

[12] M. E. Shahri, S. Balochian, H. Balochian, and Y. Zhang, "Design of fractional order PID controllers for time delay systems using differential evolution algorithm," Indian Journal of Science and Technology, vol. 7, pp. 1311-1319, 2014.

[13] D. Li, P. Ding, and Z. Gao, "Fractional active disturbance rejection control,” ISA Transactions, vol. 62, pp. 109-119, 2016.

[14] E. Koksal, "Fractional-order PID and active disturbance rejection control of nonlinear two-mass drive system," IEEE Transactions on Industrial Electronics, vol. 60, pp. 3806-3813, 2013.

[15] J. Song, J. Lin, L. Wang, X. Wang, and X. Guo, "Nonlinear FOPID and active disturbance rejection hypersonic vehicle control based on DEM biogeography-based optimization," Journal of Aerospace Engineering, vol. 30, 2017.

[16] C. Nie, H. Yang, and X. Mu, "Development and trend of vacuum induction melting technology," Vacuum, vol. 52, pp. 52-57, 2015.

[17] A. Mazumder, V. Dileep Kumar, S. Sethi, and J. Mukherjee, "Continuous monitoring of temperature of electron beam heated metal evaporation surface using controlled gas purge at viewing port in a vacuum chamber," Vacuum, vol. 161, pp. 157-161, 2019.

[18] Z. Liu, Research on Temperature Control Technology of Medium Frequency Induction Heating Furnace, Xi'an Shiyou University, Xi'an, China, 2014.

[19] O. Biro and K. Preis, "On the use of the magnetic vector potential in the finite-element analysis of three-dimensional eddy currents," IEEE Transactions on Magnetics, vol. 25, no. 4, pp. 3145-3159, 1989.

[20] A. Oustaloup, La Commande CRONE: Commande Robuste d'Ordre Non Entier, Hermès, Paris, France, 1991.

[21] A. Oustaloup, X. Moreau, and M. Nouillant, "The CRONE suspension," Control Engineering Practice, vol. 4, no. 8, pp. 1101-1108, 1996.

[22] A. Oustaloup, J. Sabatier, and X. Moreau, "From fractal robustness to the CRONE approach," ESAIM: Proceedings, vol. 5, pp. 177-192, 1998.

[23] I. Podlubny, "Fractional-order system and $\mathrm{PI}^{\lambda} \mathrm{D}^{\mu}$ controllers," IEEE Transactions on Automatic Control, vol. 44, no. 1, pp. 208-214, 1999.

[24] Z. Wu, L. Zhao, and L. Feng, "Intelligent vehicle control based on fractional order PID controller," Control Engineering of China, vol. 18, pp. 401-404, 2011.

[25] H. Fang, X. Yuan, and P. Liu, "Active-disturbance-rejectioncontrol and fractional-order proportional integral de-rivative hybrid control for hydroturbine speed governor system," Measurement and Control, vol. 51, pp. 192-201, 2018. 
[26] D. Xue and C. Zhao, "Fractional order PID controller design for fractional order system," Control Theory and Applications, vol. 24, pp. 771-776, 2007.

[27] M. Li, D. Li, J. Wang, and C. Zhao, "Active disturbance rejection control for fractional-order system," ISA Transactions, vol. 52, no. 3, pp. 365-374, 2013.

[28] J. Han, The Technique for Estimating and Compensating the Uncertainties: Active Disturbance Rejection Control Technique, National Defense Industry Press, Beijing, China, 2008.

[29] Z. Chen, "Some advances in linear active disturbance rejection control theory and engineering applications," Information and Control, vol. 46, pp. 257-266, 2017.

[30] J. Li, "Active disturbance rejection control: summary and prospects of research achievements," Control Theory and Applications, vol. 34, pp. 281-294, 2017.

[31] W. Yu, "Simulation and research of PID parameter tuning based on PSO," Journal of Chongqing Technology and Business University (Natural Science), vol. 37, pp. 18-23, 2020.

[32] Z. Gao, "Scaling and bandwidth-parameterization based controller tuning," in Proceedings of the American Control Conference, vol. 6, Denver, CO, USA, 2003.

[33] J. Sun, W. Xu, and B. Feng, "A global search strategy of quantum-behaved particle swarm optimization," in Proceedings of the 2004 IEEE Conference on Cybernetics and Intelligent Systems, vol. 1, Singapore, 2004.

[34] X. Zhou, H. Gao, B. Zhao, and L. Zhao, "A GA-based parameters tuning method for an ADRC controller of ISP for aerial remote sensing applications," ISA Transactions, vol. 81, pp. 318-328, 2018. 